\title{
Mudanças climáticas e/ou mudanças socioculturais na Amazônia Legal
}

As mudanças climáticas e socioculturais vêm gerando variações climáticas em várias regiões além de influenciar na dinâmica do ciclo hídrico das bacias no bioma amazônico. Essas mudanças desencadeiam reações de ajustes nos ciclos hidrológicos, assim o estudo propõe estabelecer conexões entre o funcionamento biológico da floresta e o clima produzidos naturalmente e/ou pela ação antrópica (socioculturais), considerando como variáveis ambientais a temperatura, precipitação e emissão de $\mathrm{CO} 2$ e como variáveis socioculturais as queimadas e o desmatamento progressivo e por corte raso. Metodologia: $\mathrm{O}$ estudo considerou, apenas, o bioma amazônico brasileiro, com recorte temporal de janeiro de 2004 a janeiro de 2016. Os dados foram coletados nos bancos de dados do INPE, INMET, NOAA, IBGE, PRODES e DETER. Resultados e discussão: Foi observada correlação entre a densidade populacional e desmatamento, justificado basicamente por uma "nova" lógica de ocupação vinculada ao grande empreendimento - frente pioneira de expansão sobre o território. O corte raso possui correlações inversas com a temperatura média máxima, e a razão de densidade populacional por área. Correlações positivas foram observadas com a precipitação total e a ocorrência das queimadas e a elevação de $\mathrm{CO} 2$. Obtiveram-se correlações positivas na elevação de $\mathrm{CO} 2$ e a razão de densidade populacional por área. As variáveis de mudanças climáticas apresentaram correlações positivas nas médias de temperatura máximas com o desmatamento progressivo e na razão de densidade populacional por área, a concentração de $\mathrm{CO} 2$ e principalmente com a ocorrência das queimadas. Fracas correlações e inversas com o desmatamento por corte raso e uma forte correlação inversa com a quantidade de precipitação. Considerações Finais: Deve-se atentar a retirada da cobertura vegetal no bioma Amazônia, em conjunto com as práticas de antropização socioculturais de cunho pirogênicas observadas no período de estudo. Demonstram haver correlações diretas e inversas e que estão fortemente relacionadas com o cenário econômico-social. Esses fatores reúnem o conjunto com potencial de contribuir na ampliação na ocorrência de eventos climáticos extremos como da estiagem (secas) e inundações impacta diretamente no ciclo hidrológico da Região Amazônica.

\section{Climate change and/or sociocultural changes in the Legal Amazon}

Climate and socio-cultural changes have produced climatic variations in several regions, such as influencing the dynamics of the water cycle in basins in the Amazonia biome. Based on recent climate events, those changes might be affecting the hydrological cycle in the tropical areas, therefore, the study proposes to establish connections between the biological functioning of the forest and the climate produced naturally and/or by the anthropic (sociocultural) activity, considering three environmental variables: temperature, precipitation and emission of $\mathrm{CO} 2$, and as sociocultural variables: man-made fires and the deforestation by clearcutting and progressive cut. Methodology: The study considered only the Brazilian Amazonian biome, with a 2-year long time frame, starting in January of 2004 to January of 2016. The data required for the study was collected from INPE, INMET, NOAA, IBGE, PRODES, and DETER databases. Results and discussion: A correlation was observed between demographic density and deforestation which can be justified by a capitalistic approach for the use of the land - a pioneer of expansion over the territory. The clearcutting technique has inverse correlations with the maximum average temperature, and the demographic density. Positive correlations were among annual averages rainfall values, the occurrence of fires and the elevation of $\mathrm{CO} 2$. Positive correlations were obtained from $\mathrm{CO} 2$ concentration and the demographic density. The climate change variables showed positive correlations among maximum temperature averages, progressive cut deforestation, demographic density rates, $\mathrm{CO} 2$ concentration and mainly with the occurrence of fires. There were low and inverse correlations among clearcutting deforestation and other variables, but a strong inverse correlation with the amount of precipitation. Conclusion: It is important to keep track of the deforestation of the Amazon biome that is nurtured by socio-cultural anthropological practices such as criminal fires. The study observed that there were direct and inverse correlations that were strongly related to the socio-economic scenario. Therefore, extreme climatic events such as droughts and flooding in the Brazilian Amazon rainforest can relate to the negative interaction between society and the natural environment.

Keywords: Climate; Amazon; Sociocultural; Droughts; Floods.

Topic: Meteorologia, Climatologia e Mudanças Climáticas

Reviewed anonymously in the process of blind peer.
Received: 01/01/2021

Approved: 26/01/2021
Diógenes Alencar Bolwerk (ii

Universidade Federal do Tocantins, Brasil http://lattes.cnpq.br/7362195345748253 http://orcid.org/0000-0002-4601-7866 diogenes.bolwerk74@gmail.com

Marina Haizenreder Ertzogue

Universidade Federal do Tocantins, Brasil http://lattes.cnpq.br/8782125323284470

http://orcid.org/0000-0002-7888-9888 marinahe@hotmail.com
Referencing this:

BOLWERK, D. A.; ERTZOGUE, M. H.. Mudanças climáticas e/ou mudanças socioculturais na Amazônia Legal. Revista Ibero Americana de Ciências Ambientais, v.12, n.1, p.202-213, 2021. DOI: http://doi.org/10.6008/CBPC2179-6858.2021.001.0017 


\section{INTRODUÇÃO}

As mudanças climáticas e socioculturais observadas no bioma amazônico vêm gerando variações climáticas como deficiências e/ou excesso de chuvas em várias regiões além de influenciar na dinâmica do ciclo hídrico das bacias. Nesse contexto os eventos climáticos associados a estas mudanças, assumem importância no cotidiano da sociedade, seja pela frequência e intensidade que se manifesta, ou pela vulnerabilidade socioambiental que expõe as comunidades envolvidas (MARENGO et al., 2011).

As mudanças climáticas desencadeiam reações de ajustes nos ciclos hidrológicos que ainda necessitam de mais estudos para serem plenamente estabelecidos. Porém, se sabe que, ao passo que as mudanças ocorrem, os ciclos hidrológicos se aceleram em busca dos ajustes e alteram a expressão do regime hídrico (NOBRE et al., 2008). Dentre as consequências das alterações climáticas na região amazônica, é importante destacar que a própria oferta e disponibilidade do recurso água, em grande parte, dependente da manutenção do equilíbrio do clima.

O ciclo anual das chuvas e a variação das vazões dos rios variam entre as bacias e a variabilidade, quando associada aos fenômenos climáticos, podem gerar anomalias, como secas e enchentes (MARENGO, 2008). Por isso que os riscos derivados das mudanças climáticas sejam naturais ou antropogênicas, isoladas ou em conjunto, configuram-se em ameaças à floresta amazônica e a perda da floresta é uma ameaça para o clima.

A diminuição na cobertura vegetal vem sendo considerada uma grave consequência da ampliação das atividades econômicas. Nesse processo os avanços no aumento da densidade demográfica, do desmatamento, da agricultura, da atividade de extrativismo vegetal, da mineração, da abertura de áreas para pastagem dos grandes empreendimentos, estão relacionados com as alterações importantes nos fluxos e ciclos biogeoquímicos e atmosféricos (ALARA et al., 2006). Observa a existência de relações com as alterações nos fluxos do carbono e nitrogênio (ANDREAE et al., 1997) da fertilidade do solo, perda da biodiversidade, degradação do solo, aumento da desertificação (NEILL, 2001), além das mudanças no ciclo hidrológico e nas relações entre a evapotranspiração e a precipitação na Amazônia (NÓBREGA et al., 2005; MARENGO, 2006).

Outro aspecto importante a considerar sobre as variabilidades e mudanças no sistema climático, sejam elas naturais ou antropogênicas, é o aumento na emissão e concentração de gases do efeito estufa na atmosfera (ARAÚJO, 2013). O cenário em construção parece poder afetar a dinâmica dos ecossistemas amazônicos, reduzindo sua capacidade de absorver o carbono da atmosfera, aumentando a temperatura à superfície, modificando o ciclo hidrológico regional, influenciando os padrões espaciais de umidade do solo e a ocorrência de eventos extremos (secas e enchentes) e consequentemente, afetando a reciclagem de precipitação (ROCHA et al., 2015).

Ainda deve-se considerar a ocorrência da evapotranspiração que existe em função da disponibilidade de energia e água e diminuição, principalmente, pela alteração da concentração de $\mathrm{CO}_{2}$ na atmosfera, que acaba desencadeando outras consequências, como por exemplo, afetando o desempenho das plantas (NOBRE et al., 2007). Outro fator são as concentrações de partículas de aerossóis e gases traço, que afetam 
os mecanismos naturais de uma série de processos atmosféricos na região amazônica (ARTAXO et al., 2005). Os fatores antrópicos, não menos importante, também impactam no regime hídrico amazônico, a região desde o Século XIX, com o ciclo do extrativismo vegetal da seringueira passa por ordenamento territorial imbuído por uma política nacional de ocupação da região (MARTINS, 1975). Sendo que o conjunto destes fatores possui capacidade de produzir alterações importantes no regime hídrico principalmente nas áreas tropicais.

Somando-se a fatores como a umidade do ar, cujo comportamento se reflete na possibilidade de viabilizar ou inviabilizar queimadas, uma vez que, se reduzida no ambiente, aumenta a inflamabilidade da vegetação segundo Ray (2005). Na mesma linha a temperatura do ar, que quando se eleva, promove elevação da probabilidade de uma fonte de ignição resultar em queimada. Pelo fato das queimadas estarem intimamente associadas às alterações no uso do solo e também serem responsáveis por emissões significativas de partículas de aerossóis para a atmosfera (ARTAXO et al., 2005) acabam produzindo alterações no conjunto de compostos orgânicos voláteis, oxigenados e gases de efeito estufa que interferem na formação dos núcleos de condensação de nuvens (NCN) (ANDREAE et al., 1997) e consequentemente no mecanismo de formação de nuvens e precipitação gerando importantes implicações sobre o ciclo hidrológico de áreas tropicais e sobre o clima em nível local, regional e global (ARTAXO et al., 2002).

As queimadas, bem como os desmatamentos estão associadas ao modelo civilizatório e desenvolvimento em curso para a região, visto que a expansão da prática capitalista, da propriedade privada, da lavoura, da pastagem, da mecanização cria o espaço do tempo "moderno". Este modelo de empreendimento econômico e estabelece o valor econômico sobre a terra e o trabalho (MARTINS, 2009). A região possui baixa densidade demográfica (IBGE, 2010) sendo de tal forma que seus impactos são reduzidos, mas o modelo de exploração e ocupação predatória do solo contribui significativamente para o ciclo do regime hídrico amazônico.

Nesse sentido, o estudo propõe contribuir com o estabelecimento das conexões entre o funcionamento biológico da floresta e o clima, uma vez que, as mudanças climáticas estão alterando os processos básicos de funcionamento do ecossistema amazônico (ARTAXO et al., 2014).

Assim, buscou-se determinar como são evidenciadas as correlações entre os fatores de mudança climáticos produzidos naturalmente e/ou pela ação antrópica (socioculturais) com as alterações do regime hídrico na região amazônica. Considerando como variáveis ambientais a temperatura, precipitação e emissão de $\mathrm{CO}_{2}$ e como variáveis socioculturais as queimadas e o desmatamento progressivo e por corte raso.

\section{METODOLOGIA}

\section{Área de estudo}

O presente trabalho foi realizado na parcial do território brasileiro contido no bioma amazônico, que abrange os estados do Pará, Amazonas, Amapá, Acre, Rondônia, Roraima, Maranhão, Tocantins e Mato Grosso, remetendo a área de aproximadamente $4.196 .943 \mathrm{~km}^{2}$, da área total do bioma, de 6 milhões de km² 
IBGE (2004). Foi escolhido o recorte temporal de janeiro de 2004 a janeiro de 2016, totalizando 12 anos de dados referentes a todas as variáveis do estudo.

\section{Coleta de dados}

\section{Detecção de focos de queimadas}

Os dados pertencentes às áreas de queimadas, referentes aos anos de 2004 a 2016, foram coletados do banco BDQueimadas do INPE (INPE, 2017).

\section{Dados de temperatura e precipitação}

Os dados de temperatura e precipitação foram retirados do banco de dados do Instituto Nacional de Meteorologia (INMET, 2017), coletados e agrupado por estados, para os anos de 2004 a 2016.

\section{Concentração de $\mathrm{CO}_{2}$ atmosférico}

A informação sobre a quantidade de $\mathrm{CO}_{2}$ expressa em partes por milhão (ppm), foi obtido a partir das informações disponibilizadas pelo NOAA (National Oceanic and Atmospheric Administration) (NASA, 2017) com creditados a ESRL (Earth System Research Laboratory) referentes aos anos de 2004 a 2016.

\section{Dados de população e área dos estados}

Os dados de área física dos estados brasileiros pertencentes ao estudo foram coletados no site do Instituto Brasileiro de Geografia e Estatística (IBGE, 2010). Já a população de cada estado, foi obtida do censo de 2010 e das estimativas anuais de população do IBGE de 2011 até 2016.

\section{Desmatamento por corte raso e progressivo}

Para determinar as médias anuais de desmatamento por corte raso e desmatamento progressivo, foram obtidos dados referentes aos anos de 2004 a 2016, disponibilizados pelo Instituto Nacional de Pesquisas Espaciais (INPE), por meio de dois sistemas operacionais, o Programa de Cálculo do Desflorestamento da Amazônia-PRODES e o Sistema de Detecção de Desmatamento em Tempo Real-DETER.

\section{Agrupamento e organização dos dados}

\section{Queimadas}

Os 1.048.576 pontos de queimada coletados foram agrupados em número de ocorrência de focos por quilômetro quadrado de área $\left(\mathrm{km}^{2}\right)$ por mês. Os valores resultantes mensais foram agrupados em ocorrências por estado e somados em valores anuais por quilômetro quadrado de área $\left(\mathrm{km}^{2}\right)$.

\section{Temperatura e Precipitação}

Dos dados de temperatura foram captados desde 2004 até 2016, em 68 sensores, gerando 10.547 
pontos de amostra de valores em graus centígrados (으). Foram calculados os valores médios das temperaturas máximas obtidas dentro de cada mês para cada ano. E os dados foram agrupados para calcular a média de temperatura máxima anual.

Os dados de precipitação representam 741 dias de chuvas onde 68 sensores produziram 10.457 amostras de dados. Esses valores foram agrupados em valores de milímetros cúbicos de precipitação hídrica $\left(\mathrm{mm}^{3}\right)$ por mês em cada localidade do sensor. Destes valores mensais, foram divididos por estados e calculados o valor acumulado anual de $\mathrm{mm}^{3}$ de precipitação hídrica anual.

\section{Concentração de $\mathrm{CO}_{2}$}

Os dados de gás carbônico captados, diariamente e representados em médias mensais, sendo que para os valores ausentes, foram adotadas médias interpoladas. Dos valores mensais da fração molar de $\mathrm{CO}_{2}$, ou seja, o número de moléculas de $\mathrm{CO}_{2}$ em cada milhão $(\mathrm{ppm})$ de moléculas secas ar (vapor de água removido), foi calculada a média aritmética entre os doze meses de cada ano, resultando em um valor que representa a média anual de emissão de $\mathrm{CO}_{2}$.

\section{Densidade demográfica}

Para calcular a estimativa de densidade populacional e área dos estados, foram captados os dados existentes no banco de dados do IBGE (IBGE, 2010). Na densidade populacional foram utilizados dados do censo demográfico do ano de 2010, a partir desses dados foram utilizadas as estimativas de população fornecidas pelo IBGE. Esses dados de população foram divididos pela área total do estado, resultando em um quociente de densidade populacional por $\mathrm{km}^{2}$.

\section{Desmatamento por corte raso e progressivo}

Foram coletados 20.766 pontos de desmatamento por corte raso no banco de dados do PRODIS e 12.921 pontos de desmatamento por corte progressivo, no DETER, que foram organizados em valores de área desmatada e expressos em quilômetros quadrados $\left(\mathrm{km}^{2}\right)$ e agrupados em ocorrências no mesmo estado por ano.

\section{Tabela de valores primários agrupados e normalizados}

Os valores produzidos foram agrupados a partir dos dados primários gerando 13.731 linhas de dados para um dos eixos de informação colhida. Desta tabela primária, os valores foram agrupados e normalizados para eliminação das diferenças de escala considerando a natureza da informação para grupo. Após o tratamento estes dados foram analisados estatisticamente para obtenção das correlações.

Tabela 1: dados agrupados por descritor e ano, normalizados para aplicação dos testes de correlações.

\begin{tabular}{llllllll}
\hline Ano & $\begin{array}{l}\text { Densidade } \\
\left(\mathrm{pop} / \mathrm{km}^{2}\right)\end{array}$ & $\begin{array}{l}\text { Prec. } \\
\mathrm{mm}^{3}\end{array}$ & $\begin{array}{l}\text { Temp. Média } \\
\text { Máxima }\left({ }^{\circ} \mathrm{C}\right)\end{array}$ & $\begin{array}{l}\text { Desmatamento } \\
\text { Corte Raso }\end{array}$ & $\begin{array}{l}\text { Desmatamento } \\
\text { Corte Progressivo }\end{array}$ & $\begin{array}{l}\text { Queimadas } \\
\left(\text { Focos } / \mathrm{km}^{2}\right)\end{array}$ & $\begin{array}{l}\mathrm{CO}_{2} \\
\mathrm{Ppm}\end{array}$ \\
\hline 2004 & 4.627 & 167.248 & 33.397 & $1.61 \mathrm{E}-05$ & $1.57 \mathrm{E}-02$ & 0.132 & 380.432 \\
2005 & 4.639 & 169.867 & 32.943 & $1.63 \mathrm{E}-04$ & $1.52 \mathrm{E}-02$ & 0.127 & 379.797 \\
\hline
\end{tabular}




\begin{tabular}{|c|c|c|c|c|c|c|c|}
\hline 2006 & 4.729 & 186.889 & 32.586 & $2.04 \mathrm{E}-04$ & $1.54 \mathrm{E}-02$ & 0.073 & 381.896 \\
\hline 2007 & 4.761 & 166.431 & 32.910 & $1.13 \mathrm{E}-04$ & $1.57 \mathrm{E}-02$ & 0.028 & 383.792 \\
\hline 2008 & 4.793 & 179.544 & 32.562 & $1.18 \mathrm{E}-04$ & $1.59 \mathrm{E}-02$ & 0.049 & 385.604 \\
\hline 2009 & 4.853 & 180.877 & 32.707 & $3.72 \mathrm{E}-05$ & $1.61 \mathrm{E}-02$ & 0.041 & 387.430 \\
\hline 2010 & 4.963 & 159.433 & 33.282 & $2.14 \mathrm{E}-05$ & $1.62 \mathrm{E}-02$ & 0.078 & 389.899 \\
\hline 2011 & 5.073 & 180.466 & 32.756 & $2.63 \mathrm{E}-05$ & $1.63 \mathrm{E}-02$ & 0.036 & 391.652 \\
\hline 2012 & 5.138 & 162.650 & 32.905 & $3.02 \mathrm{E}-05$ & $1.64 \mathrm{E}-02$ & 0.067 & 393.853 \\
\hline 2013 & 5.298 & 182.179 & 32.785 & $2.82 \mathrm{E}-05$ & $1.66 \mathrm{E}-02$ & 0.036 & 396.521 \\
\hline 2014 & 5.366 & 169.385 & 32.781 & 4.11E-05 & $1.67 \mathrm{E}-02$ & 0.070 & 398.648 \\
\hline 2015 & 5.432 & 144.090 & 33.461 & 4.47E-05 & $1.68 \mathrm{E}-02$ & 0.209 & 400.834 \\
\hline 2016 & 5.496 & 157.641 & 33.483 & 5.17E-05 & $1.70 \mathrm{E}-02$ & 0.205 & 404.209 \\
\hline
\end{tabular}

\section{Análise de dados}

\section{Definição metodológica para as correlações}

Para definição das correlações entre as variáveis, foi aplicado testes de correlação paramétricos e não-paramétricos. Para correlações paramétricas foi aplicada a metodologia de correlação de Pearson $\left(r^{2}\right)$. Já para correlações não-paramétricas, foi aplicado o teste de Kendall (tau), que consiste em um procedimento estatístico para medir a correlação e a associação não-paramétrica para comparar variáveis não relacionadas.

\section{RESULTADOS E DISCUSSÃO}

\section{Correlações socioculturais}

Os resultados das variáveis relacionadas às mudanças socioculturais demonstradas na Tabela 1, como as queimadas são apresentadas por quilômetro quadrado e apontam que após o segundo ano, ocorreu decréscimo, seguido de uma média estacionária, com oscilações variando sobre esta média temporal, no entanto, nos últimos anos houve um acréscimo significativo, chegando, quase o dobro do primeiro ano.

Ao observar que no ano de 2015 houve um aumento extraordinário de focos de incêndios, onde basicamente no ano de 2016 manteve-se este cenário. O curioso é o ano de 2015, apresentou os menores índices pluviométricos, nesse sentindo constata-se que devido a peculiaridades do clima amazônico, os incêndios são recorrentes nos períodos de baixa precipitação e baixa umidade.

O balanço de radiação e o ciclo hidrológico também podem ser afetados indiretamente pelas emissões de queimadas, via alterações na microfísica e na dinâmica de formação de nuvens (UHL et al., 1990), em função de uma maior disponibilidade de núcleos de condensação de nuvem e gelo na atmosfera, os quais promovem mudanças no espectro de gotas de nuvem e estabilização termodinâmica.

Quando aplicado o coeficiente de Kendall (tau) para testar a correlação entre densidade populacional e queimadas no bioma amazônico, observou-se que não há correlação. Assim, procurou na literatura a fundamentação para explicar o fenômeno apresentado pelos dados tratados e normalizados. Até o final dos anos 1960, à lógica de ocupação do território amazônico dava-se exclusivamente pelo estímulo da migração, a partir do final da década de 1960, esse modelo de ocupação tornou-se secundário, pois à “nova" lógica imprimi uma ocupação vinculada principalmente ao grande empreendimento capitalista e altamente mecanizado (MARTINS, 1975). 
Sendo assim, o modelo civilizatório é conduzido pela inserção de uma "nova" lógica do modo de produção capitalista, que tem como características a predação da natureza e da implementação de relações sociais capitalistas e no uso indiscriminado, exploratório e predatório de todas as fontes de recursos naturais, num mecanismo acelerado de mercantilização da natureza e suas potencialidades e na consecução da exploração do trabalho humano (MARTINS, 2009).

Ao tratar-se dos impactos do desmatamento, percebeu-se que os resultados obtidos no período de 2004 a 2016, o desmatamento por corte raso concentrou os maiores valores no início do período de coleta dos dados e manteve valores decrescentes e consecutivos por todo o período restante do estudo. $A$ análise não-paramétrica revelou que o corte raso possui correlações significativas e inversas com a temperatura média máxima, e a razão de densidade populacional por área. Também foi demonstrado haver correlações positivas com a precipitação total e a ocorrência das queimadas e a elevação de $\mathrm{CO}_{2}$.

$\mathrm{Na}$ análise do coeficiente de Kendall (tau) ao mesmo passo que demonstra ter correlações significativas, os valores muito próximos de zero, revelam a baixa intensidade destas relações. E assim sugerem a necessidade de avaliar outros fatores, como os socioculturais, para elucidar o entendimento da ocorrência do corte raso. Nepstad et al. (2001) e Meyfroidt et al. (2013) afirmam a necessidade de entender os processos dinâmicos ligados à economia e políticas públicas para explicar à lógica dessas ações antrópicas na Região Amazônica.

Estabelecer a relação entre o desmatamento e o modelo de desenvolvimento para a região, implica na compreensão de como o território está sendo ocupado, isto é, como a política nacional de créditos impulsiona os agentes sociais no ordenamento e uso do solo. Logo, relacionar a forma de apropriação do território e como se dá o desmatamento da floresta é observar como à lógica capitalistas está organizada (MARTINS, 2009).

Para Phillips (2002) e Nepstad et al. (2004) é necessário que se considerem as complementares entre a cobertura vegetal, o ciclo hídrico e a manutenção do sistema climático. Uma vez que a cobertura vegetal removida, além de diminuir a capacidade de armazenar e capturar $\mathrm{CO}_{2}$, ainda agrava o cenário, pois a emissão de gases potencializa efeitos lesivos tanto ao clima, quanto para a biodiversidade. Neste sentido as mudanças climáticas serão ao mesmo tempo consequências e promotoras de novos eventos climáticos e rearranjos em busca de reequilibrar o sistema climático.

Já os resultados obtidos no período de 2004 a 2016, sobre o desmatamento progressivo. Ao contrário da expressão por corte raso, teve um comportamento crescente em todo o período do estudo. $A$ análise nãoparamétrica dos dados revela que o desmatamento progressivo desenvolve fortes correlações positivas com a elevação de $\mathrm{CO}_{2}$ e com a razão de densidade populacional por área. Esses resultados concordam com os resultados de Ferreira (2005) que afirmam que as altas taxas da perda da floresta pelo desmatamento em Mato Grosso existem e podem ser explicadas pelo modelo de desenvolvimento do estado que concentra na grande propriedade privada ( $\geq 1.000$ ha) a sua lógica desenvolvimentista que utiliza em larga escala a mecanização dos processos produtivos em comparação com outros estados da Região Amazônica.

Os resultados da estimativa de densidade populacional por quilômetro quadrado existente na 
Amazônia nos anos de 2004 a 2016 demonstram que a densidade populacional estimada é variável ao longo do período de estudo, apresentando um crescimento equilibrado nos últimos anos.

Para Becker (1998) a tendência é um processo de desconcentração da população das regiões metropolitana, e maior participação nas cidades de até 100.000 habitantes, observando crescimento elevado da população em núcleos pequenos de até 20.000 habitantes.

A análise das correlações revelou que a densidade populacional apresenta forte correlação com as variáveis de corte progressivo e $\mathrm{CO}_{2}$. A relação é de 1 para 1, com taxa de regressão linear de $100 \%$. Assim, estabelece um coeficiente de correlação "perfeita". Os dados apresentados corroboram com Araújo (2013), Ferreira (2005), Marengo (2008) e Betts et al. (2004) afirmam que o desmatamento é resultado do aumento das atividades econômicas.

\section{Variáveis ambientais}

As variáveis de mudanças climáticas apresentaram na Tabela 1 resultados, como as médias de temperatura máximas obtidas no período de 2004 a 2016, demonstrando que houve variabilidade interanual em todo o período do estudo, todavia menos pronunciada nos primeiros anos do estudo. As variabilidades se elevaram a partir de 2008, com um pico importante em 2010 e outro e 2015, no final do período em estudo. Na análise da distribuição das frequências (Figura1-B) percebe que as médias das variações da temperatura sugerem duas modas, o que poderia ser interpretado como uma provável alteração na condição ambiental no período.

A aplicação de probabilidade pelo teste $Q-Q$ plot (Figura 1-C) aponta tendência de perfil de linearidade no período estudado, sugere que os estudos de regressão linear seriam pouco eficientes na explicação do fenômeno. No entanto, os valores da correlação de Pearson desmontaram haver correlações estatísticas significativas diretas da temperatura com o desmatamento progressivo, a razão de densidade populacional por área, a concentração de $\mathrm{CO}_{2}$ e principalmente com a ocorrência das queimadas. Também apresenta uma fraca correlação e inversa com o desmatamento por corte raso e uma forte correlação inversa com a quantidade de precipitação.
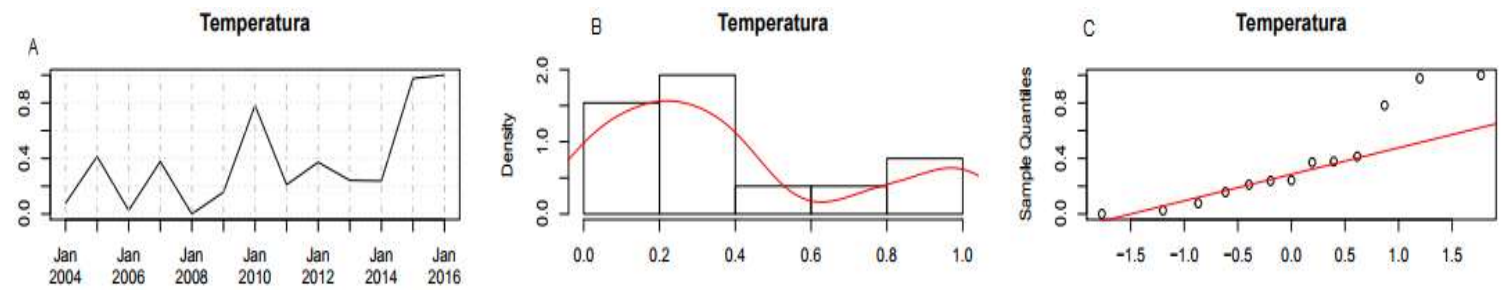

Figura 1: Média de temperaturas máximas na Amazônia no período de 2004 a 2016.

Os valores de temperatura comparados pelo teste não-paramétrico de correlação de Kendall, revelam haverem correlações relativamente fracas para maioria dos parâmetros verificados conforme é observado. Mas a correlação entre a temperatura e a precipitação apresentou valores fortes, porém inversos. Marengo $(2006 ; 2007)$ afirma o aquecimento pode se dar por causa de fatores naturais, como o aquecimento das águas do Atlântico Sul, observados desde 1950, ou a fatores antropogênicos como desmatamentos e o 
efeito da urbanização das cidades, ou a uma combinação destes fatores.

Ao considerar que existem tendências na elevação de temperatura (Figura 1-A), em conjunto com a observação da diminuição da precipitação na região percebidos na Figura 2-A. Convém destacar que os maiores valores foram observados na época sazonal do inverno, ou seja, entre os meses de julho a agosto, considerada estação de transição do período chuvoso para o seco na região da Amazônia. Essa ocorrência é importante porque o aumento destes valores, a tomar pela correlação fortemente negativa, observada com a precipitação pode interferir nos processos de evapotranspiração, formação de nuvens, consequentemente interferir nos ciclos hídricos.

Os resultados de precipitação total anual e volume de chuvas por milímetro cúbico no bioma Amazônico (Figura 2-A) no período no recorte temporal do estudo, demostram que nos primeiros anos a precipitação anual apresentou variações ao longo dos anos com um decréscimo significativo nos últimos anos. A análise do histograma (Figura 2-B) mostra a representação da frequência da precipitação de chuvas em ordem crescente, com a distribuição da frequência da densidade populacional ao longo tempo. A representação em teste de Q-Q Plot (Figura 2-C) representa a força da correlação de precipitação do período coletado.
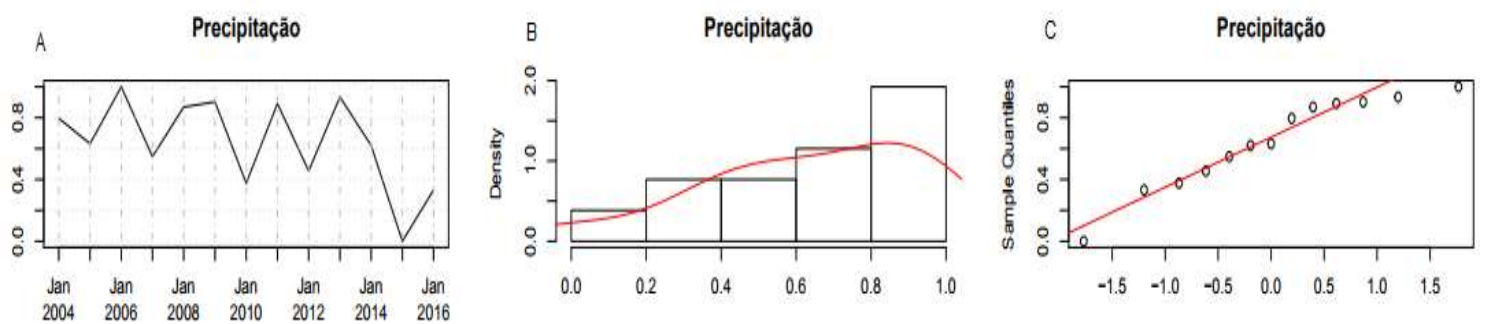

Figura 2: Taxas médias anuais de precipitação na Amazônia no período de 2004 a 2016.

Levando-se em consideração as taxas médias anuais de precipitação, Rocha et al. (2015) afirma que cerca de $20 \%$ da precipitação na Amazônia decorre da evapotranspiração local, as mudanças no sistema climático estão relacionadas às variações naturais e as ações antropogênicas, afetando a dinâmica dos ecossistemas amazônicos, e modificando o ciclo hidrológico regional. Vasconcellos (2015) em avaliação da suscetibilidade da vegetação do sul do Amazonas nos anos de 2004 e 2005, durante a época da seca, observou que em anos de maior seca, o maior percentual de focos de calor ocorreu em células com baixa precipitação mensal e altas temperaturas médias e umidade relativa inferior a $65 \%$.

Observa-se que os anos de 2008 e 2009, apresentaram taxas de precipitação com comportamento semelhantes, nesse sentido Marengo (2009) afirma que o ano 2009 a Região Amazônica apresentou chuvas e enchentes muito intensas, sendo causado pelo fenômeno La Niña. Algumas regiões a situação tão crítica que o governo disponibilizou recursos para as pessoas desabrigadas, atividades diárias da população foram modificadas, atingindo a atividade pesqueira e o acesso às escolas. A abundante vegetação amazônica libera grande quantidade de vapor de água através da evapotranspiração o que permite a região também participar de maneira importante (25 a 35\%) no ciclo de reciclagem da precipitação de água (TRENBERTH, 2007; NÓBREGA et al., 2005). 
Os resultados de emissão de $\mathrm{CO}_{2}$ (Figura 3) descrevem o aumento que vem ocorrendo em todo o período do estudo. Os valores de menores concentrações foram observados no início do estudo e os valores mais elevados ao final do período. A medida desta elevação (curva de Keeling) é abordada por vários cientistas com muita cautela, devido à divergência na probabilidade e natureza dos efeitos no meio ambiente (MARENGO et al., 2012; FEARNSIDE, 2005; 2009).
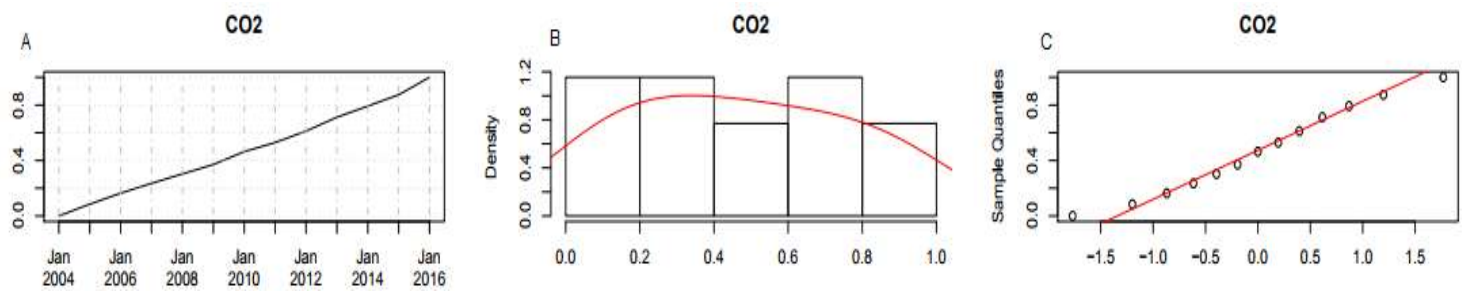

Figura 3: Emissão de $\mathrm{CO}_{2}$ na Amazônia no período de 2004 a 2016.

Nesse sentido, as correlações testadas no trabalho expõem que existem relações positivas e de grande intensidade (coeficiente de Pearson) com o desmatamento progressivo e a razão de densidade populacional por área. Na mesma linha as correlações mantiveram intensidades menores, mas mantendo o sentido de positividade quando correlacionados pelo teste de Kendall. Os resultados do trabalho complementam os achados na literatura, pois entre 1970 a 2004, citam a elevação nas emissões de Gases de Efeito Estufa (GEE) em escala global, sendo os principais agentes o setor de transporte e a indústria. A emissão de $\mathrm{CO}_{2}$, fruto das queimadas na região é controverso, por causa da dificuldade inerente em determinar quanto de $\mathrm{CO}_{2}$ é liberado na atmosfera pela queima da biomassa (NOBRE et al., 2007).

Estima que na Amazônia a quantidade máxima de carbono liberada está entre 0,24 e 0,42 TgC/ano (teragramas, ou milhões de toneladas, de carbono por ano). A estimativa que nas florestas tropicais em todo o mundo, este valor aproximasse, de 2TgC/ano (HOUGHTON et al., 1991). A Amazônia contribui com 12\% a 21\% dessa quantidade, porém, apesar de haver consenso em que a emissão de gás carbônico aumenta o efeito estufa, não existe dados conclusivos sobre o aumento de temperatura, relações demonstradas pela baixa intensidade das correlações apresentadas no trabalho. Considerar a ideia de que, se as emissões de gases carbônicos continuam crescendo, haverá sensíveis mudanças climáticas na Terra, é ainda uma afirmação incerta. As pesquisas ainda estão em níveis especulativos e sua real atuação no processo de alteração climática permanece carente de comprovações.

\section{CONCLUSÕES}

Considerando a temática da água e a sustentabilidade na Amazônia, onde as alterações no sistema climático da região refletem sobre as alterações no regime hídrico. A ocorrência de estações de secas e enchentes se definiu com diferentes níveis de relação entre os fatores ambientais e socioculturais.

Pode-se inferir que no período de 2004 a 2016, o aumento da densidade populacional por área na região amazônica, confirma a tendência crescente de ocupação humana no território. No entanto, é de se considerar que a ocorrência das ações antrópicas neste bioma está mais direcionada ao modelo civilizatório e desenvolvimento econômico do que a própria densidade populacional. 
Neste cenário, considerando que as variações nos valores dos componentes parametrizados, as análises e teste das hipóteses, não foram de natureza linear (Q-Q-Plot), e não corresponderam em significativo coeficiente de Pearson $(p>0,001)$, nem satisfatório coeficiente de determinação $\left(R_{2}\right)$. A interpretação de Kendall se mantém efetiva considerando o grau de concordância entre os pontos coletados e a ordenação de coletas durante os anos, a repetitividade de resultados, e a variação das correlações de Kendall.

A constatação da associação de correlações entre os fatores influenciadores do efeito estufa, do desmatamento e da variação do regime hídrico que em conjunto promovem as alterações climáticas observadas na região. Observou-se que a associação inversa da quantidade de precipitação com as ocorrências de queimadas e a elevação da temperatura média máxima no período do estudo soma-se com as associações positivas como a elevação da temperatura média máxima e com a elevação de $\mathrm{CO}_{2}$, bem como o aumento na razão de densidade populacional por área e o desmatamento por corte progressivo. Fatos que demonstram a importância das atividades antrópicas, no saldo da modificação produzida dos forçantes do sistema climáticos na região amazônica.

Nesta linha, deve-se atentar a retirada da cobertura vegetal no bioma Amazônia, em conjunto com as práticas de antropização cultural de cunho pirogênicas observadas no período de estudo. Demonstram haver correlações diretas e inversas e que estão fortemente relacionadas com o cenário econômico. Neste contexto, o avanço sobre o território estabelece uma "nova sociabilidade" fundada na economia de mercado que introduz a modernidade tecnológica que tem como características a exploração predatória como forma de ocupação. E esses fatores reúnem o conjunto com potencial de contribuir na ampliação na ocorrência de eventos climáticos extremos como da estiagem (secas) e inundações impacta diretamente no ciclo hidrológico da Região Amazônica.

\section{REFERÊNCIAS}

ALARA, L. L.; HOLLAND, E. A.; ARTAXO, P.; CAMARGO, P. B.; MARTINELLI, L. A.. Land use and expanding industrialization are changing nitrogen deposition in Brazil. Biogeochemistry (in press), 2006. DOI: https://doi.org/10.1590/18071929/agriambi.v19n1p70-76

ANDREAE, M. O.; CRUTZEN, P. J.. Atmospheric aerosols: biogeochemical sources and role in atmospheric chemistry. Science, v. 276, p. 1.052-1.058, 1997.

ARAÚJO, H. J. B.; OLIEVIRA, L. C. V. S. S.; CORREIA, M. F.. Danos provocados pelo fogo sobre a vegetação natural em uma floresta primária no estado do acre, Amazônia brasileira. Ciência Florestal, v.23, n.2, p.297-308, 2013. DOI: http://dx.doi.org/10.5902/198050989276

ARTAXO, P.; MARTINS, J. V.; YAMASOE, M. A.; PROCÓPIO, A. S.; PAULIQUEVIS, T. M.; ANDREAE, M. O.; GUYON, P.; GATTI, L. V.; LEAL, A. M. C.. Physical and chemical properties of aerosols in the wet and dry season in Rondônia, Amazonia. Journal of Geophysical Research, v.107, 2002. DOI: https://doi.org/10.1029/2001JD000666

ARTAXO, P.; GATTI, L. V.; LEAL, A. M. C.; LONGO, K. M.; FREITAS, S. R.; LARA, L. L.; PAULIQUEVIS, T. M.; PROCÓPIO, A. S.; RIZZO, L. V.. Química atmosférica na Amazônia: a floresta e as emissões de queimadas controlando a composição da atmosfera amazônica. Acta Amazônica, v.35, n.2, p.185-196, 2005. DOI: https://dx.doi.org/10.1590/S0044-59672005000200008

ARTAXO, P.; DIAS, M. A. F. S.; NAGY, L.; LUIZÃO, F. J. CUNHA, H. B.; QUESADA, C. A. N.; MARENGO, J. A.; KRUSCHE, A. Perspectivas de pesquisas na relação entre clima e o funcionamento da floresta amazônica. Ciência e Cultura, v.66, n.3, p.41-46, 2014. DOI: http://dx.doi.org/10.21800/S0009-67252014000300014

BECKER, B. K.. A Especificidade do Urbano na Amazônia: Desafios para políticas Públicas Consequentes. Estudo elaborado para a Secretaria de Coordenação dos Assuntos da Amazônia. Legal - Ministério do Meio Ambiente. Rio de Janeiro: Ministério do Meio Ambiente, 1998.

BETTS, R. A.; COX, P. M.; COLLINS, O. P.; HUNTINGFORD, C.; JONES, C. D.. The role of ecosystem-atmosphere interactions in simulated Amazonian precipitation decrease and forest dieback under global change warming. Theoretical and Applied Climatology, v.78, p.157-175, 2004. DOI: http://doi.org/10.1007/s00704-004-0050-y

FEARNSIDE, P. M.. Deforestation in Brazilian Amazonia: 
history, rates and consequences. Conservation Biology, v.19, n.3, p.680-688, 2005. DOI: http://doi.org/10.1111/i.15231739.2005.00697.x

FEARNSIDE, P. M.. Global warming in Amazonia: impacts and Mitigation. Acta Amaz, v.39, n.4, 2009. DOI: https://doi.org/10.1590/S0044-59672009000400030

FERREIRA, A. P.. Cruzando fronteiras disciplinares: um panorama dos estudos migratórios. Rio de Janeiro: Revan, 2005.

HOUGHTON, J. T.; JENKINS, G. J.; EPHRAUMS, J. J.. IPCC Climate Change: the IPCC scientific assessment. Cambridge: Cambridge University Press, 1991.

IBGE. Instituto Brasileiro de Geografia e Estatística. Censo Demográfico 2010. IBGE, 2010.

IBGE. Instituto Brasileiro de Geografia e Estatística. Bioma. IBGE, 2004.

INMET. Instituto Nacional de Meteorologia. Clima. INMET, 2017.

INPE. Instituto Nacional de Pesquisas Espaciais. Queimadas: monitoramento de focos. INPE, 2017.

MARENGO, J. A.. Interdecadal variability and trends of rainfall across the Amazon basin. Theoretical and Applied Climatology, n.78, p.79-96, 2004. DOI: http://doi.org/10.1007/s00704-004-0045-8

MARENGO, J. A.. On the hydrological cycle of the Amazon basin: a historical review and current state-of-the-art. Revista Brasileira Meteorologia, v.21, n.3, p.1-19, 2006.

MARENGO, J. A.. Mudanças climáticas globais e seus efeitos sobre a biodiversidade: caracterização do clima atual e definição das alterações climáticas para o território brasileiro ao longo do século XXI. 2 ed. Brasília: Ministério do Meio Ambiente, 2007.

MARENGO, J. A.. Água e mudanças climáticas. Estudos Avançados, São Paulo, v.22, n.63, p.83-96, 2008. DOI: https://doi.org/10.1590/S0103-40142008000200006

MARENGO, J. A.; TOMASELLA, J.; ALVES, L. M.; SOARES, W. R.; RODRIGUEZ, D. A.. The drought of 2010 in the context of historical droughts in the Amazon region. Geophysical Research Letters, v.38, p.1-5, 2011. DOI: https://doi.org/10.1029/2011GL047436

MARENGO, J. A.; CHOU, S. C.; KAY, G.; ALVES, L. M.; PESQUERO, J. F.; SOARES, W. R.; SANTOS, D. C.; LYRA, A. A.; SUEIRO, G.; BETTS, R.; CHAGAS, D. J.; GOMES, J. L.; BUSTAMANTE, TAVARES, T.. Development of regional future climate change scenarios in South America using the Eta CPTEC/ HadCM3 climate change projections: climatology and regional analyses for the Amazon, São Francisco and the Parana River Basins. Climate Dynamics, v.38, p.1829-1848, 2012. DOI: http://doi.org/10.1007/s00382-011-1155-5

MARTINS, J. S.. Frente pioneira: contribuição para uma caracterização sociológica. In: MARTINS, J. S.. Capitalismo e tradicionalismo no Brasil: estudos sobre as contradições da sociedade agrária no Brasil. São Paulo: Pioneira, 1975. p.4350.

MARTINS, J. S.. O tempo da fronteira: retorno à controvérsia sobre o tempo histórico da frente de expansão e da frente pioneira. In: MARTINS, J. S.. Fronteira: a degradação do Outro nos confins do humano. 2 ed. São Paulo: Contexto, 2009. p.131-179. DOI: https://doi.org/10.1590/ts.v8i1.86141

MEYFROIDT, P.; LAMBIN, E.; HEINZ, K.; HERTEL, T. W.. Globalization of land use: distant drivers of land change and geographic displacement of land use. Current Opinion in Environmental Sustainability, v.5, p.438-444, 2013. DOI: http://dx.doi.org/10.1016/j.cosust.2013.04.003

NASA. National Aeronautics and Space Administration. Metadados. NASA, 2017.

NEPSTAD, D. C.; CARVALHO, G.; BARROS, A. C.; ALENCAR, A.; CAPOBIANCO, J. P.; BISHOP, J.; MOUTINHO, P.; LEFEBVRE, P.; SILVA JUNIOR, U. L.; PRINTS, E.. Road paving, fire regime feedbacks, and the future of Amazon forests. Forest Ecology and Management, v.154, p. 395-407, 2001. DOI: http://doi.org/10.1016/S0378-1127(01)00511-4

NEPSTAD, D. C.; LEFEBRE, P.; SILVA, U. L.; TOMASELLA, J.; SCHLESINGER, P.; SOLÓRZANO, L.; MOUTINHO, P.; RAY, D.; BANITO, J. G.. Amazon drought and its implications for forest flammability and tree growth: a basin-wide analysis. Global Change Biology, v.10, p.704-717, 2004. DOI: https://doi.org/10.1111/i.1529-8817.2003.00772.x

NOBRE, C. A.; SAMPAIO, G.; SALAZAR, L.. Mudanças climáticas e Amazônia. Ciências e Cultura, v.59, n.3, 2007.

NOBRE, C. A.; OLIVEIRA, G, S.. Mudanças climáticas. Emília Wanda Rutkowski. In: Mudanças Climáticas e Mudanças Socioambientais Globais: reflexões sobre alternativas de futuro, Brasília: UNESCO, 2008.

NÓBREGA, R. S.; CAVALCANTI, E. P.; SOUZA, E. P.. Reciclagem de vapor d'água sobre a América do Sul utilizando Reanálises do NCEP-NCAR. Revista Brasileira Meteorologia, v.20, p.253-262, 2005.

RAY, D.; NEPSTAD, D.; MOUTINHO, P.. Micrometeor-ological and canopy controls of fire susceptibility in forested Amazon landscape. Ecological. Applications, v.15, p.1664-1678, 2005. DOI: https://doi.org/10.1890/05-0404

ROCHA, V. M.; CORREIA, F. W. S.; FONSECA, P. A. M.. Reciclagem de precipitação na Amazônia: um estudo de revisão. Revista Brasileira de Meteorologia, v.30, n.1, p.5970, 2015. DOI: https://doi.org/10.1590/0102-778620140049

TRENBERTH, K. E.; JONES, P. D.. Observations: Surface and Atmospheric Climate Change. New York: Cambridge University Press, 2007.

UHL, C.; KAUFFMAN, J. B.. Deforestation, fire susceptibility, and potential tree responses to fire in the eastern Amazon. Ecology, p.71, p. 437-449, 1990. DOI: http://doi.org/10.2307/1940299

A CBPC - Companhia Brasileira de Produção Científica (CNPJ: 11.221.422/0001-03) detém os direitos materiais desta publicação. Os direitos referem-se à publicação do trabalho em qualquer parte do mundo, incluindo os direitos às renovações, expansões e disseminações da contribuição, bem como outros direitos subsidiários. Todos os trabalhos publicados eletronicamente poderão posteriormente ser publicados em coletâneas impressas sob coordenação da Sustenere Publishing, da Companhia Brasileira de Produção Científica e seus parceiros autorizados. Os (as) autores (as) preservam os direitos autorais, mas não têm permissão para a publicação da contribuição em outro meio, impresso ou digital, em português ou em tradução. 